\title{
Robust forecast aggregation: Fourier $L_{2} E$ regression
}

\section{Daniel Cross ${ }^{1}$ | Jaime Ramos ${ }^{2}$ | Barbara Mellers ${ }^{3}$ | Philip E. Tetlock ${ }^{3}$ | David W. Scott ${ }^{1}$}

\author{
${ }^{1}$ Department of Statistics, Rice University, \\ Houston, TX, USA \\ ${ }^{2}$ Department of Statistics, Indiana \\ University, Bloomington, IN, USA \\ ${ }^{3}$ Department of Psychology, University of \\ Pennsylvania, Philadelphia, PA, USA
}

\section{Correspondence}

Daniel Cross, Department of Statistics, Rice University, Houston, TX 77251, USA. Email: dmcross0@gmail.com

\section{1 | INTRODUCTION}

The problem of political prediction is a serious endeavor for students of history. The modern media is replete with experts and pundits who offer their judgment of the likelihood of future events. Tetlock (2005) summarizes a long-term study of the accuracy of such forecasts, and found surprisingly poor performance by such experts. The Intelligence Advanced Research Projects Activity (IARPA) initiated a competition among teams organized by universities to evaluate the accuracy of aggregate forecasts over thousands of individuals for hundreds of shortand medium-term scenarios, called individual forecasting problems (IFP). The Good Judgment Team (GJT) was by the far the most successful in terms of accuracy of predictions, based upon the smaller Brier score. The inner workings of the GJT are described Ungar et al. (2012). A subset of the forecasters was identified as consistently providing low Brier scores. These so-called "super forecasters" truly performed in a consistently high manner. Tetlock and Gardner (2015) provide a summary of the overall project.

Among the dozens of algorithmic approaches for analyzing the reams of data from the thousands of individuals who provided forecasts for every question, two algorithms were consistently in the top two spots at the forecast aggregation task. The individuals' forecasts were not just yes/no, but actual probability vectors for the two to five options available in each IFP scenario. Individuals were free to update their forecasts at any time, as new information was acquired. A variation of logistic regression eventually took top honors. Close behind was a robust regression approach that began with a minimum distance criterion (integrated 
squared error) applied to regression called $L_{2} E$ developed by Scott (2001). The new robust regression approach was initially the work of Ramos (2014), and then modified and extended in the final year by the first and last authors of this report; continued work has narrowed the gap to almost a tie. In what follows, we discuss the statistical criteria, data preparation, and the $L_{2} E$ aggregation algorithm of the final solution.

\section{2 | BRIER SCORE CRITERION}

The quality of these aggregated forecasts for a single IFP over $N$ days was evaluated by the Brier score:

$$
\frac{1}{N} \sum_{i=1}^{N} \sum_{j=1}^{r}\left(f_{i j}-o_{j}\right)^{2},
$$

where $f_{i j}$ denotes the aggregated forecast probability of event $j$ at the end of day $i, o_{j} \in\{0=$ does not occur, $1=$ occurs $\}$ is the actual outcome of event $j$, and $r$ is the number of categories or possible choices for the question. The range of $r$ for this paper is $2-5$; in particular, our method is not limited to the binomial case of $r=2$.

Using the Brier score as an accuracy measure has several benefits and drawbacks. Some of the benefits include ease of understanding, continuity, and discouraging extremization. However, there are several drawbacks as well. Some examples include a disconnect from the discreteness of reality, and the ability to attempt to "game" the system.

"Gaming" the system can be attempted by choosing only easy IFPs or by submitting few forecasts for the more difficult IFPs and many on the easier IFPs (see GJP, 2015b). Something that may at first seem like a good idea is extremizing all forecasts to 0 or 1 ; that is, pushing forecasts away from the average value of $1 / r$, where $r$ is the number of categories. This is actually a poor choice because the Brier score punishes extremizing harshly, and the randomness of the real world makes oracle forecasting impossible. By observing the successful strategies of the top individual forecasters, the optimum choice seems to be to give conservative estimates, then extremize as soon as the solution seems certain. At the beginning of each year after the first 10 IFPs are concluded, the top $40 \%$ of forecasters are chosen and labeled "Top Individuals." This list of top individuals is updated after the conclusion of each IFP to maintain the top $40 \%$ of forecasters by Brier score. The total number of forecasters for the final year was 1,799 , of which 629 were designated as top individual forecasters at one time or another.

\section{3 | VARIANCE-STABILIZING TRANSFORMATION}

Given that the forecasts are probabilities $p \in[0,1]$ and there are only a few decimal places an "expert" typically uses when providing forecasts, we can assume that $p$ only takes values on a finite set $\left\{0, \frac{1}{n}, \ldots, \frac{n-1}{n}, 1\right\}$. Therefore, let $P^{*} \sim \operatorname{binom}(n, \xi)$, (where the binomial probability is given by $\xi$ to avoid confusion with the symbol $p$ );

$\operatorname{Prob}\left[P^{*}=p^{*}\right]=\left(\begin{array}{c}n \\ p^{*}\end{array}\right) \xi^{p^{*}}(1-\xi)^{n-p^{*}}, p^{*} \in\{0,1, \ldots, n\}$.

Let $P=g\left(P^{*}\right)=P^{*} / n$. Then

$$
\begin{aligned}
\operatorname{Prob}[P=p] & =\operatorname{Prob}\left[g\left(P^{*}\right)=p\right] \\
& =\operatorname{Prob}\left[P^{*}=n p\right], p \in\{0,1 / n, \ldots, 1\} .
\end{aligned}
$$

Also,

$$
E[P]=E\left[g\left(P^{*}\right)\right]=\frac{1}{n} E\left[P^{*}\right]=\xi .
$$

Now setting $\mu=\xi$,

$$
\sigma^{2}=\operatorname{var}[P]=\operatorname{var}\left[g\left(P^{*}\right)\right]=\frac{1}{n^{2}} \operatorname{var}\left[P^{*}\right]=\frac{\xi(1-\xi)}{n} .
$$

Assuming there exists a relationship between the mean, $\mu$, and variance, $\sigma^{2}$, such as

$$
\sigma^{2}=\gamma(\mu)
$$

one can use a two-term Taylor series approximation to solve for a transformation $T(P)$ that gives an approximately constant variance using the formula $T^{\prime}(\mu)=(\gamma(\mu))^{-1 / 2}$. Thus the variance-stabilizing transformation (VST) solves the differential equation $T^{\prime}(p)=(p(1-p) / n)^{-1 / 2}$, or

$$
T(p)=-2 \sqrt{n} \arctan \sqrt{\frac{1-p}{p}} .
$$

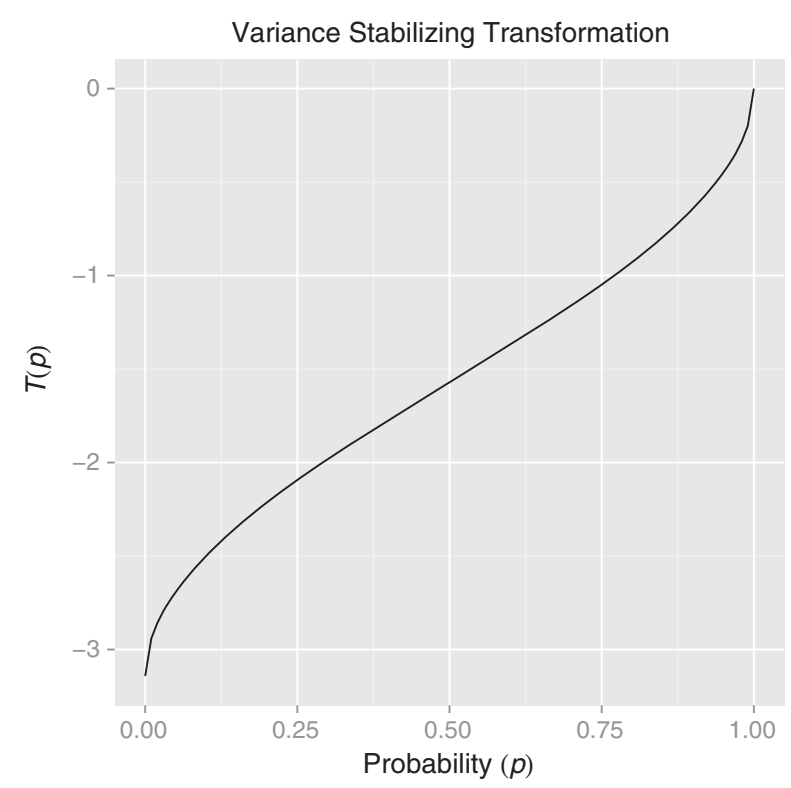

FIGURE 1 Variance-stabilizing transformation with $n=1$ 
Ramos (2014) derived this form of the transformation, which invokes the odds ratio. The original and equivalent form, derived by Anscombe (1948), was essentially $\sin ^{-1} \sqrt{p}$, which involves only $p$ itself.

One should note that this transformation is well defined for all $p \in[0,1]$ and $\operatorname{var}[T(P)]=1$ for any $n$. We choose $n=1$ for the remainder of this paper. Looking at Figure 1, forecast probabilities near the extreme values of 0 or 1 are 'stretched out' towards the endpoints of the interval $[-\pi, 0]$. This somewhat forces forecasts to be more conservative and therefore takes advantage of the squared error in the Brier score criterion.

As an example of the benefits of the VST, we may exam(see Section 5).

\section{4 | $L_{2} E C R I T E R I O N$ SOLUTIONS}

Our robust approach to the estimation task is to use a minimum distance criterion called $L_{2} E$, which has many desirable properties (see Scott, 2001). These are discussed in the two sections below.

\section{1 | $L_{2}$ Ecriterion}

Using the parametric density $f(x \mid \theta)$ to model the true (unknown) density g, an estimate of the $L_{2}$ criterion

$$
\begin{aligned}
L_{2}(\theta) & =\int(f(x \mid \theta)-g(x))^{2} d x \\
& =\int f(x \mid \theta)^{2} \mathrm{~d} x-2 E\left[f(X \mid \theta]+\int g(x)^{2} \mathrm{~d} x\right.
\end{aligned}
$$

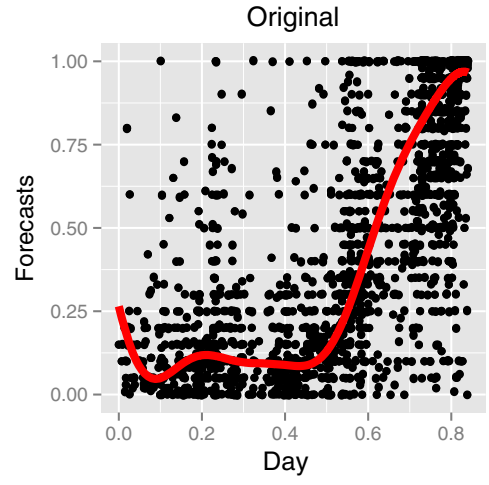

Original Residuals
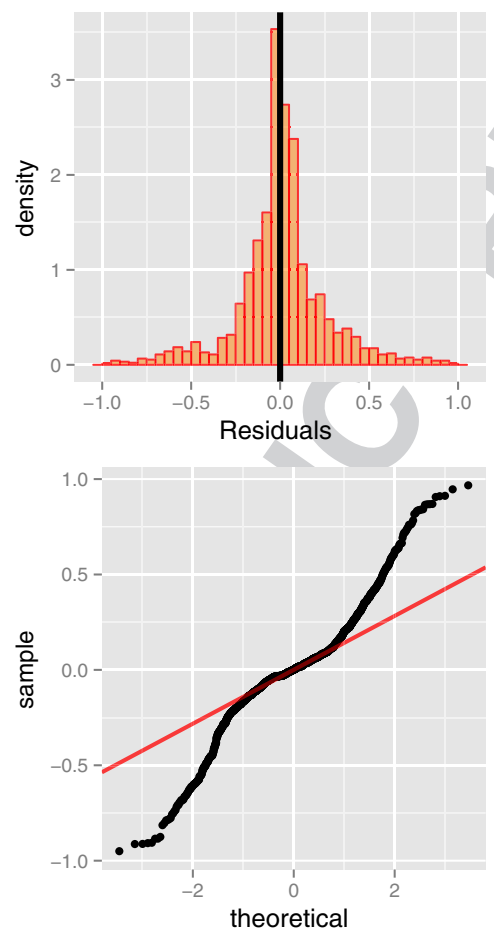

IFP ID = 1412-0a

With VST
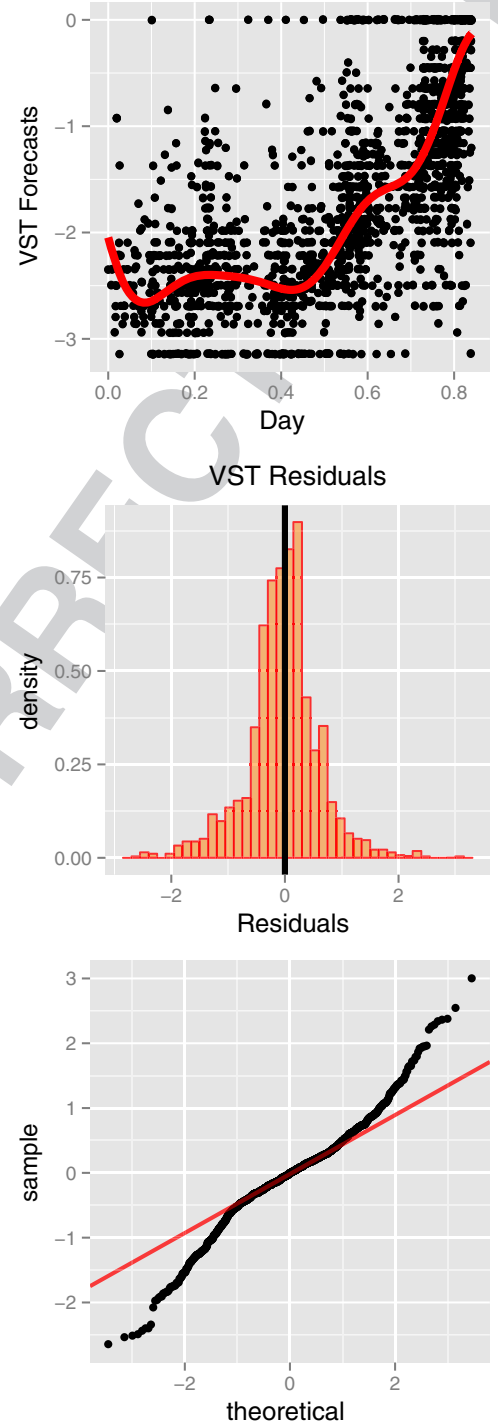

Post-VST
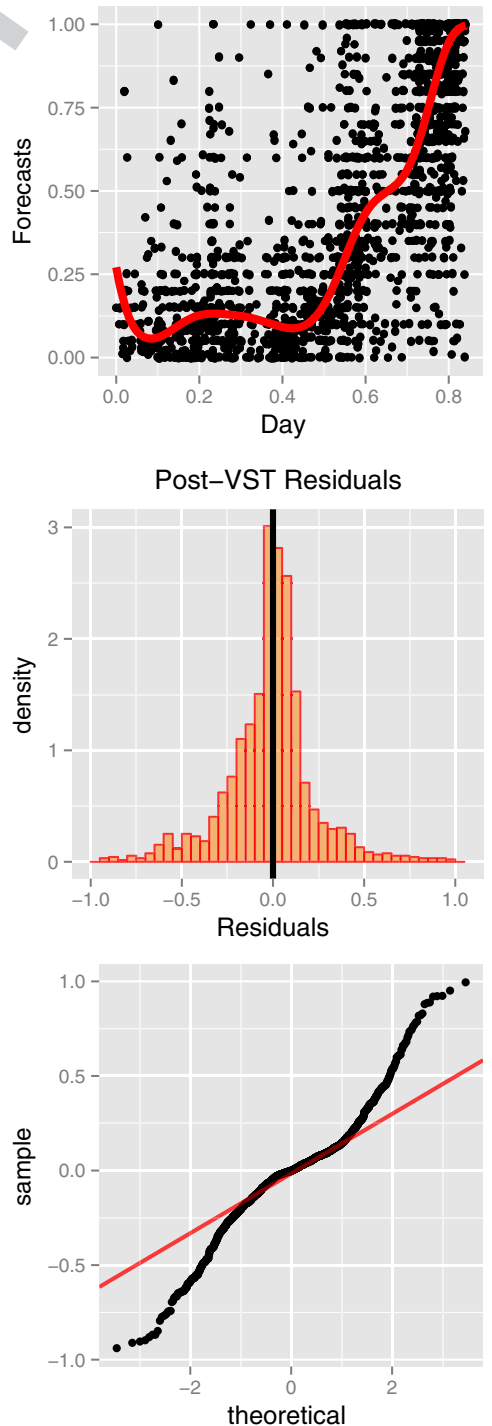

FIGURE 2 Differences in VST and non-VST regressions. Column 1 is the regression without any transformation of the data. Column 2 is the regression with the VST $T(p)$ applied to forecasts $p$. Column 3 is the regression in column 2 with the data and regression with $T^{-1}(p)$ applied for all forecasts $p$ [Colour figure can be viewed at wileyonlinelibrary.com] 
is given by the fully data-based estimator

$$
\hat{\theta}_{L_{2} E}=\underset{\theta}{\operatorname{argmin}}\left[\int f(x \mid \theta)^{2} \mathrm{~d} x-\frac{2}{n} \sum_{i=1}^{n} f\left(x_{i} \mid \theta\right)\right],
$$

which may be minimized numerically. Note that $\frac{1}{n} \sum f\left(x_{i} \mid \theta\right)$ provides an unbiased estimator of $E[f(X \mid \theta)]$, whereas $\int g(x)^{2} \mathrm{~d} x$ does not depend on $\theta$ and may be omitted.

There is a variation of $L_{2} E$ called partial $L_{2} E\left(w L_{2} E\right)$, which was introduced by Scott (2004), that can take into account the largest portion, $w$, of the data that are fitted by the density model. This strategy attempts to ignore the portion, $1-w$, that does not come from the model. The $w L_{2} E$ criterion adds one parameter, $w$, as follows:

$$
L(\theta, w)=\underset{\theta, w}{\operatorname{argmin}}\left[w^{2} \int f(x \mid \theta)^{2} \mathrm{~d} x-2 w E(f(X \mid \theta)],\right.
$$

where $w$ is the "partial" parameter that estimates the portion of the data that is captured by the model. By accounting for the presence of outliers, $w L_{2} E$ outperforms the standard $L_{2} E$ by a significant margin in many cases. The only drawback is that the extra parameter adds computing time. The density $f(x \mid \theta)$ is often chosen to be the normal density. In the context of regression, the model's random variables are the residuals from the regression curve, $m_{\theta}(x)$. Criterion 3 can be extended for use in regression by letting the random variable $X$ be the residuals, $\epsilon$, of the regression with zero mean and variance $\sigma^{2}$. Therefore, combining a normal residual model with $w L_{2} E$, the regression criterion is

$$
\hat{L}(\theta, \sigma, w)=\frac{w^{2}}{2 \sqrt{\pi} \sigma}-\frac{2 w}{n} \sum_{i=1}^{n} \phi\left(\epsilon_{i} \mid 0, \sigma^{2}\right),
$$

where $\epsilon_{i}=y_{i}-m_{\theta}\left(x_{i}\right)$ and $\theta$ are the parameters in the regression curve $m_{\theta}(x)$. The $L_{2} E$ and $w L_{2} E$ criteria with the normal model (Equation 4) are nonconvex and may have multiple local minima. This makes the minimization of the criterion oftentimes difficult, especially with many parameters. A common quasi-Newton optimization function used in $R$ is nlminb. As with any optimization routine, nlminb requires initial values to start. With nonconvex criteria, special care is required.

To address this, several tools are available. One is the use of simulated annealing. Kirkpatrick, Gelatt, and Vecchi (1983) introduced simulated annealing first for solving discrete systems with many local minima. A problem with using simulated annealing, however, is that our domain is continuous and there are many parameters. This leads to unacceptably slow computation and sub-par results. Another option is to use 11 random starting values, choosing the median of the individual aggregation probability estimates. While taking the sample mean of these may seem like a better idea, taking the median proved the superior choice. For example, the sample mean can lead to problems when the data are bimodal, such as computing a value, post-VST, in $[0.3,0.7]$ when the individual aggregation points are clustered near 0 and 1 . While this can also happen with the median, the likelihood of this happening is lessened. Thus the median option was used, lending some extra stability to the $L_{2} E$ solutions.

Another issue was the discretization of the forecasts by the human subjects, who could only go so far when choosing probabilities. Looking at the histogram of the IFP forecasts pre- and post-VST in Figure 3, we can see the noncontinuity of the forecasts selected by the thousands of human subjects. We note that the frequency of the finer forecasts increases as the forecast values get closer to the extreme values of $-\pi$ and 0 . This raises some difficult questions pertaining to our numerical implementation of $L_{2} E$. Given that the normal model is being used, theoretically any of the "lumped" forecast points could be chosen with a $\hat{\sigma}$ value close to 0 , as shown in the next paragraph.

Consider a regression curve such that $I$ is the set of forecasts with residuals $\epsilon_{i}=0$ and $n^{\prime}=|I|>0$, then substituting the normal density into Equation 4 returns

$$
\begin{aligned}
\hat{L}(\theta, \sigma, w) & =\frac{w^{2}}{2 \sqrt{\pi} \sigma}-\frac{2 w}{n \sqrt{2 \pi} \sigma} \sum_{i=1}^{n} e^{-\epsilon_{i}^{2} / 2 \sigma^{2}} \\
& =\frac{w^{2}}{2 \sqrt{\pi} \sigma}-\frac{2 w n^{\prime}}{n \sqrt{2 \pi} \sigma}-\frac{2 w}{n \sqrt{2 \pi} \sigma} \sum_{i \in I^{c}} e^{-\epsilon_{i}^{2} / 2 \sigma^{2}} \\
& =\left(\frac{w^{2}}{2 \sqrt{\pi}}-\frac{2 w n^{\prime}}{n \sqrt{2 \pi}}\right) \frac{1}{\sigma}-\frac{2 w}{n \sqrt{2 \pi} \sigma} \sum_{i \in I^{c}} e^{-\epsilon_{i}^{2} / 2 \sigma^{2}}
\end{aligned}
$$
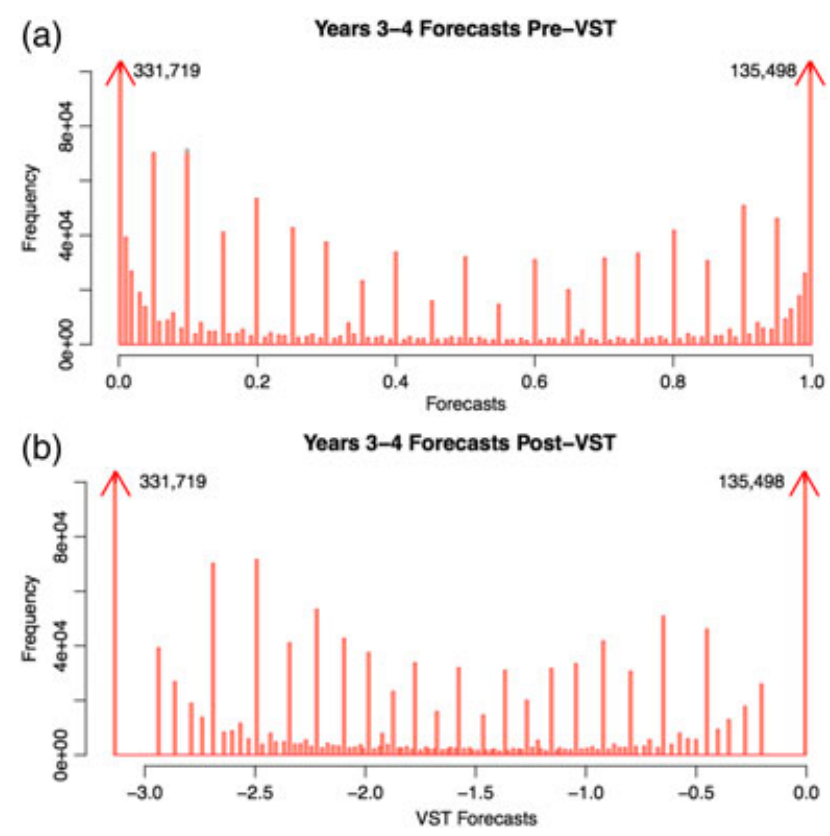

FIGURE 3 (a) Pre-VST histogram of all expert forecasts over years 3-4. (b) Post-VST histogram of all expert forecasts over years 3-4 [Colour figure can be viewed at wileyonlinelibrary.com] 


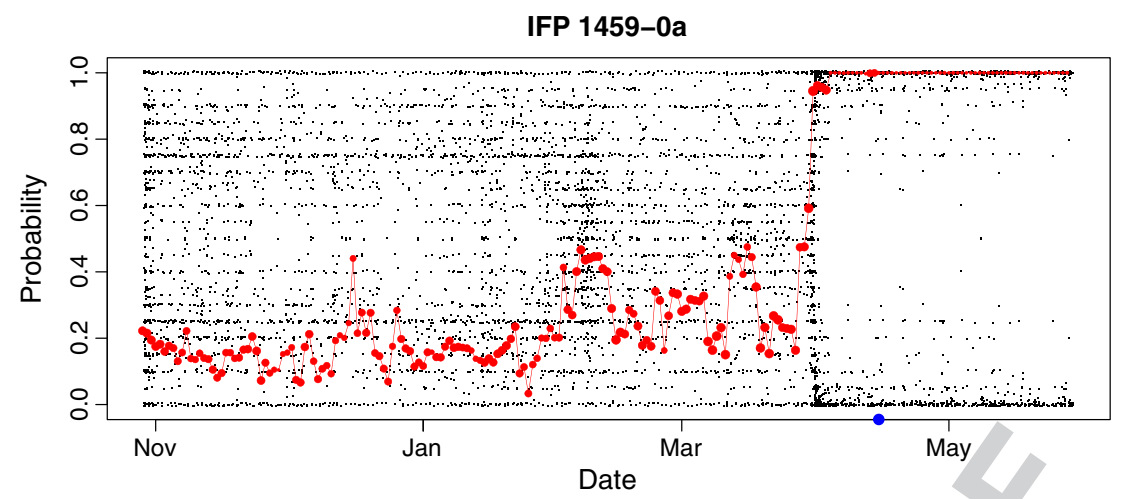

FIGURE 4 A time series plot of the aggregated forecasts, with individual forecasts indicated by small black dots. The area of each red forecast point is related to its estimated $L_{2}$ standard deviation by using the radius $0.35+\hat{\sigma}_{L_{2} E}^{1 / 2}$. After April 15 (blue dot), $\hat{\sigma}_{L_{2} E}<0.001$ [Colour figure can be viewed at wileyonlinelibrary.com]

Therefore, for any $w<\frac{n^{\prime} 2 \sqrt{2}}{n}$, the $\lim _{\sigma \rightarrow 0} \hat{L}(\theta, \sigma, w)=-\infty$. This is a feasible but uninteresting solution since $w$ is the parameter that the partial $L_{2} E$ criterion is optimized over.

To look more closely into this, consider the single IFP option 1459-0a shown in Figure 4. Figure 4 shows that the less extreme estimates before April have larger $\hat{\sigma}_{L_{2} E}$ values, as well as an example of why the median should be used over the sample mean. Later, when the forecasts reach the mode of 1 , the $\hat{\sigma}_{L_{2} E}$ values become close to 0 . This makes sense because, given two (or more) discrete points, $L_{2} E$ should select the value with the highest density and "ignore" the remainder. The fear is that this could happen to any of the individual discretization phenomena such as 0.1 , or 0.15 , depending upon the starting guesses provided to nlminb. Blurring the data by a scaled beta distribution is one method of handling this problem, which we discuss in the next section.

\section{2 | Blurring forecasts}

One method to address the issue of discreteness of forecasts posed above is to transform the data into a continuous form that retains its structure. Blurring the point at $x=$ $x_{0} \in[0,1]$ by a beta distribution is chosen because of its finite support along with the relative ease of choosing the shape of the blurring density. To keep the blurred data relevant, we chose the blurring beta density to have its mode at $x_{0}$ with width a chosen distance $h$. To be precise, the new blurred value $x 0 \prime \prime$ is sampled from a transformed beta distribution with support $\left[\max \left(0, x_{0}-h\right), \min \left(1, x_{0}+h\right)\right]$ and a mode equal to $x_{0}$. An additional constraint is needed to have the mode equal to $x_{0}$. Consider a beta $(\alpha, \beta)$ distribution, whose mode is at $(\alpha-1) /(\alpha+\beta-2)$. Then we add the constraint $\alpha+\beta=W$, for some conveniently large value of $W>2$ (we chose $W=20$ ). We insist that $W$ is large enough so that $\operatorname{beta}(\alpha, \beta)$ is unimodal, that is, $\alpha, \beta>1$. This fully specifies the beta parameters $\alpha$ and $\beta$ by solving the following set of equations:

$$
\begin{aligned}
& x_{0}=\frac{\alpha-1}{\alpha+\beta-2}, \\
& W=\alpha+\beta .
\end{aligned}
$$

Adding the constraint $\alpha+\beta=W$ lets us not only specify the mode but also the variance of the $\operatorname{beta}(\alpha, \beta)$ distribution as well. Solving Equation 5 for $\alpha$ and $\beta$ gives us

$$
\begin{aligned}
& \alpha=x_{0}(W-2)+1, \\
& \beta=W-(W-2) x_{0}-1 .
\end{aligned}
$$

Let the mapping from interval $\left[l_{1}, u_{1}\right]$ to interval $\left[l_{2}, u_{2}\right]$ be denoted by $M_{0}$, given below:

$$
M_{0}\left(x, l_{1}, u_{1}, l_{2}, u_{2}\right)=l_{2}+\frac{\left(x-l_{1}\right)\left(u_{2}-l_{2}\right)}{\left(u_{1}-l_{1}\right)} .
$$

Then the explicit steps that define the blurring of the data are given by:

- Find $x_{0}^{\prime}=M_{0}\left(x_{0}, \max \left(0, x_{0}-h\right), \min \left(1, x_{0}+h\right), 0,1\right)$.

- Blur: $x 0 \prime \prime ~ \sim ~ B e t a\left(x_{0}^{\prime}(W-2)+1, W-(W-2) x_{0}^{\prime}-1\right)$.

- Return to the original interval by $x_{b}=$ $M_{0}\left(x_{0}^{\prime \prime}, 0,1, \max \left(0, x_{0}-h\right), \min \left(1, x_{0}+h\right)\right)$.

We now treat these blurred forecasts, $x_{b}$, as the true forecast data in each of our multiple random starts. Each random start invoked new blurring and parameter guesses. This turns the discrete forecasts into continuous forecasts and solves the discreteness issue. In Figure 5 we show examples of the beta distributions used for blurring near the VST boundary $-\pi$ for $h=0.04$ and $W=20$. Note these beta distributions become more symmetrical away from the boundary, as shown in the right-hand frame.

The method for selecting $h$ (given $W=20$ ) used was simple minimization of the mean Brier score. The best value of $h$ was found to be approximately 0.04 for the first year (see Figure 6). 

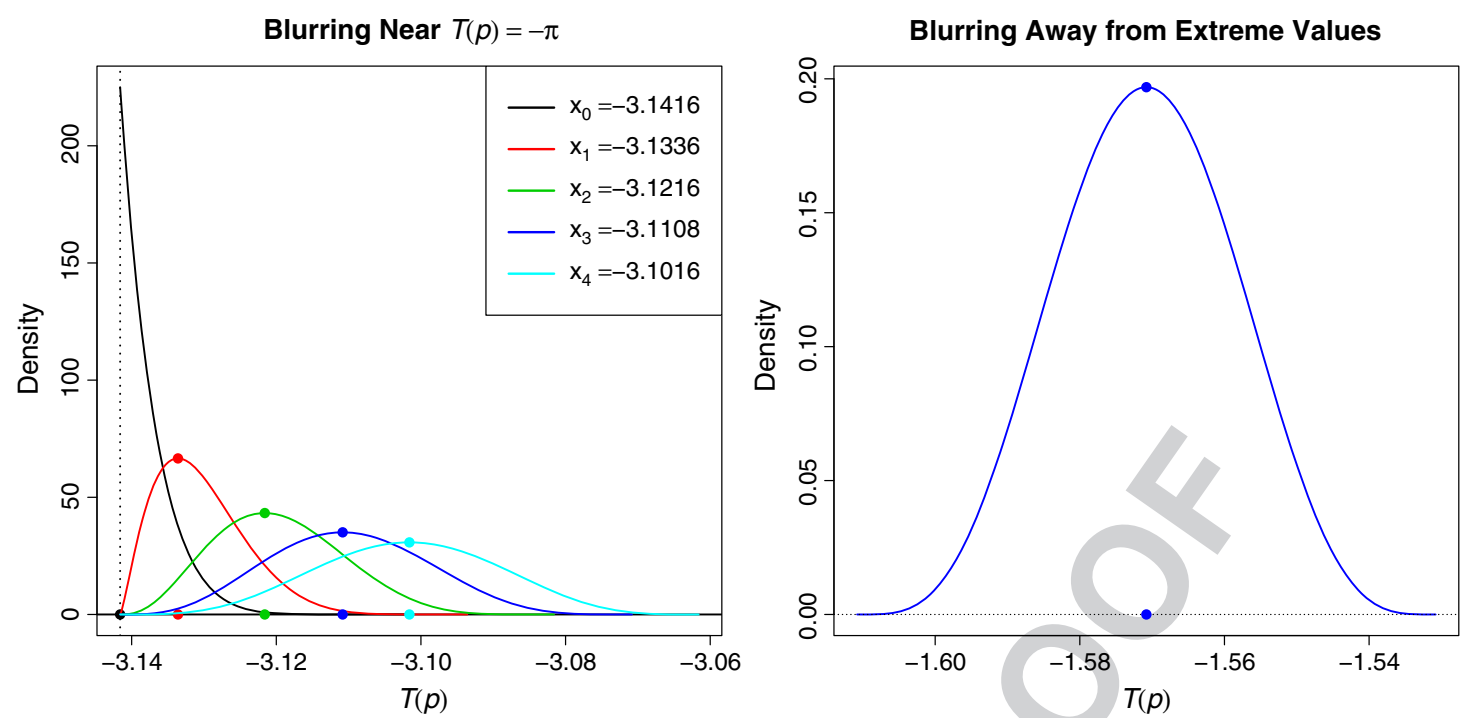

FIGURE 5 Blurring distributions examples for values close to and far from endpoints for $h=0.045$ [Colour figure can be viewed at wileyonlinelibrary.com]

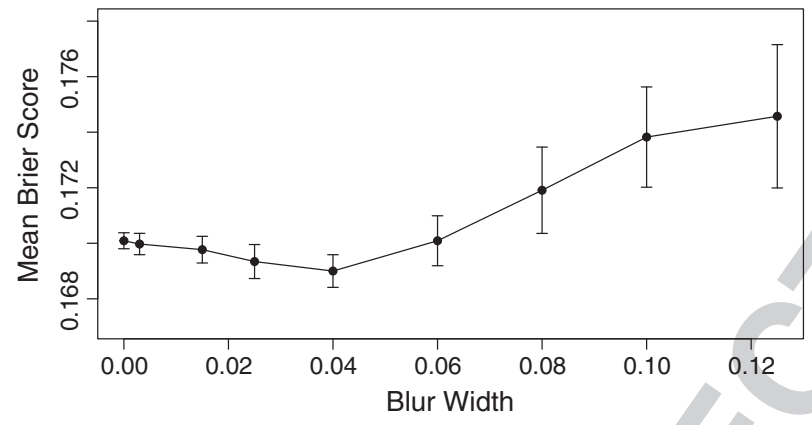

FIGURE 6 Blurring impact on Brier score for year 1 data

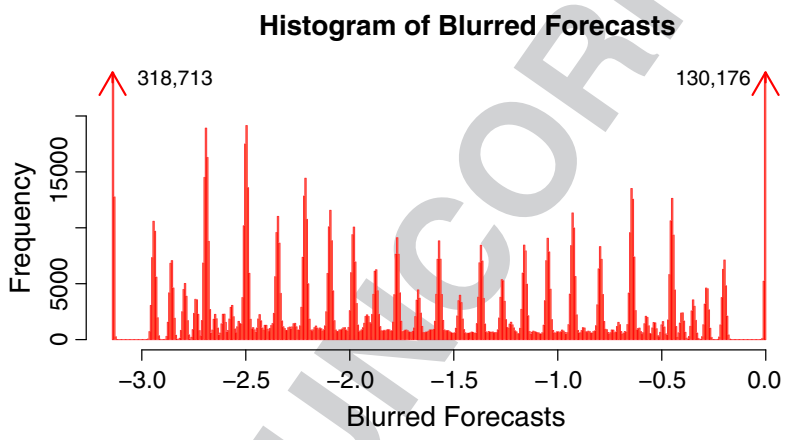

FIGURE 7 Histogram of all blurred expert forecasts over years 3-4 with $h=0.04$ and $W=20$ [Colour figure can be viewed at wileyonlinelibrary.com]

With these values of $W$ and $h$, we see a decrease in the mean Brier score by approximately 0.002 and a decease in the standard deviation by approximately 0.006 . A histogram of all probabilities from years 3 and 4 is shown in Figure 7, which should be compared to the histograms in Figure 3. The data are now continuous, which should reduce the probability of the $L_{2} E$ criterion selecting repeated discrete values.

\section{I AGGREGATION THROUGH $L_{2}$ EREGRESSION}

There are many ways to aggregate forecasts into a single value. Ungar et al. (2012) discusses some common aggregation methods including averaging, taking the median, fitting regression curves, among others. Regression of a time series is a method that makes theoretical and intuitive sense because forecasts will follow some flow through time as information becomes available and is updated. The original implementation in Ramos (2014), which scored very well, simply used linear regression over a short time horizon. To account for the often rapid change in the flow of perception of the IFPs, a regression using a truncated Fourier series can be used. A Fourier series regression is more flexible than using a linear regression. For example, one can take advantage of the useful properties of the Fourier series, such as allowing for discontinuities, while controlling the number of elements in the series to avoid overfitting. Using a truncated Fourier series regression and a normal model for the residuals, $f(\epsilon \mid \theta)=\phi\left(\epsilon \mid 0, \sigma^{2}\right)$, a truncated Fourier series can be fitted to model the time series of forecasts. The type of truncated Fourier series used to approximate a regression function $m_{\theta}(x)$ is shown in Equation 6:

$$
\hat{m}_{\theta}(x)=\frac{1}{2} a_{0}+\sum_{n=1}^{K} a_{n} \cos (2 \pi n x)+\sum_{n=1}^{K} b_{n} \sin (2 \pi n x),
$$

where $a_{0}, a_{n}$, and $b_{n}$ are the parameters to optimize over, and $K$ is a value chosen by training using the Brier score. 

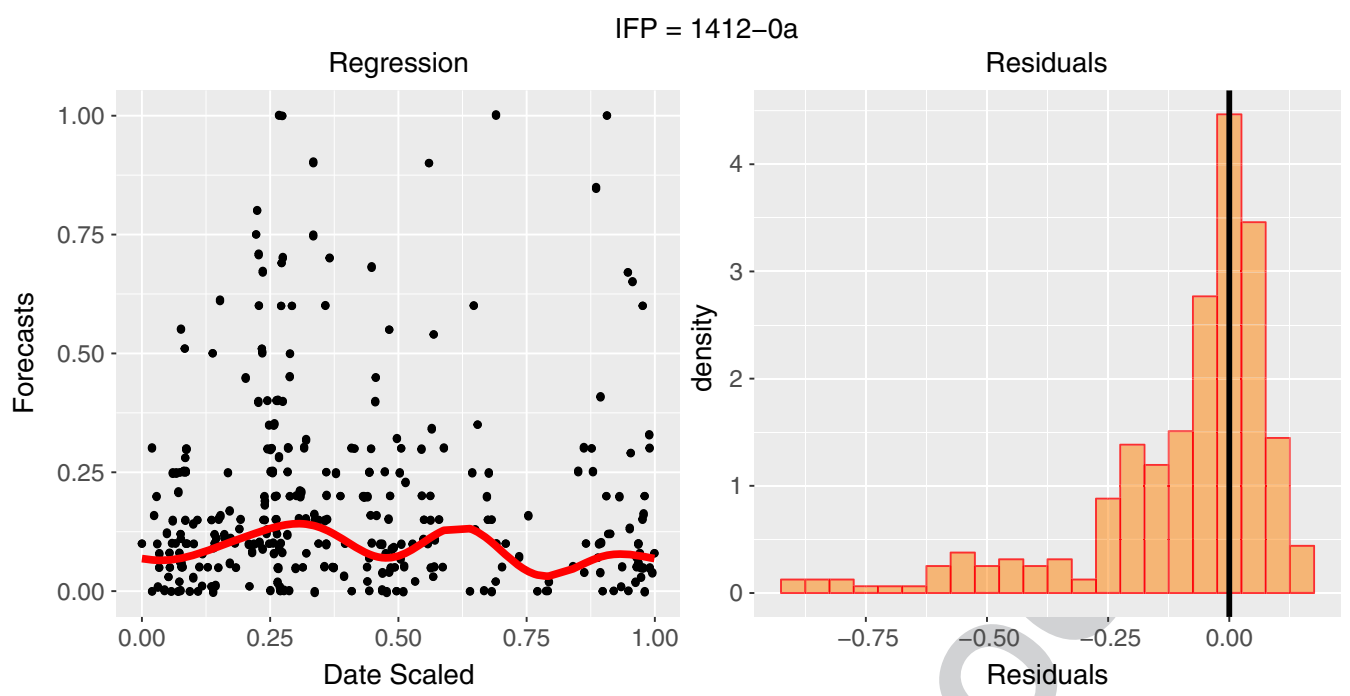

FIGURE 8 Fourier $L_{2} E$ regression over IFP 1412-0 category (a) for the final 96-hour window [Colour figure can be viewed at wileyonlinelibrary.com]

By training over all year 1 IFPs, the value $K=4$ in $m_{\theta}(t)$ and a time window of 96 hours were found to be close to optimal. Once fitted, the aggregated forecast is chosen to be the final (rightmost) regression point. For example, if the date stamps over $\left[t_{0}-96, t_{0}\right]$ hours are scaled to $[0,1]$ and a regression $m_{\theta}(t), t \in[0,1]$, is fitted to the time series, then $m_{\theta}(1)$ is the aggregated forecast at $t=t_{0}$ (see Figure 8). In this example, the above forecast aggregation would be 0.07 . Once the aggregated forecasts are found for each category (2-5 for each IFP), some type of normalization must be applied. This is discussed in the next section.

\section{6 | PROBABILITY NORMALIZATION PROCEDURE}

Given that there is no guarantee that the $r$ aggregated forecasts are proper forecasts, some normalization must be applied. The constraints that need to be met by any $r$ forecasts are

$$
0 \leq p_{i} \leq 1 \quad \text { for } i=1, \ldots, r \quad \text { and } \quad \sum_{i=1}^{r} p_{i}=1 .
$$

The most common normalization method consists of two steps: (1) adjust all values to be in $[0,1]$ by setting all values greater than 1 to 1 , and all values less than 0 to 0 ; (2) normalize by dividing each value by the sum of all values. While this method works well in our context, Ramos (2014) derived a superior method called complement normalization.

Complement normalization is an iterative process that takes advantage of the fact that one event occurring implies that other events are not occurring. Consider the binomial case $(r=2), P(A)=p_{a}, P(B)=p_{b}$. Therefore, $P(A)=1-p_{b}$, $P(B)=1-p_{a}$, and $1=P(A)+P(B)=2-p_{a}-p_{b}$. If $P(A)+P(B) \neq 1$ then $p_{a}$ and $p_{b}$ are used as estimates of complement probabilities:

$$
\begin{aligned}
& \hat{p}_{a}=1-p_{b}, \\
& \hat{p}_{b}=1-p_{a} .
\end{aligned}
$$

The complement normalization would then be

$$
\begin{aligned}
\hat{p}_{a}^{\prime} & =\frac{1-p_{b}}{2-p_{a}-p_{b}}, \\
\hat{p}_{b}^{\prime} & =\frac{1-p_{a}}{2-p_{a}-p_{b}} .
\end{aligned}
$$

The generalized form of complement normalization iteration for $r$ categories with probabilities $p_{1}, \ldots, p_{r}$ is

$$
\hat{p}_{i}^{\prime}=\left[\frac{1+(r-1) p_{i}-\sum_{j=1}^{r} p_{j}}{r-\sum_{j=1}^{r} p_{j}}\right] I_{(0, \infty]}\left(p_{i}\right),
$$

which replaces negative probabilities with zero. Equation 8 is then applied recursively on all $p_{i}^{\prime}>0$, resulting in values $p_{1, k}^{\prime}, \ldots, p_{r, k}^{\prime}$ after the $k$ th step. Let the number of feasible probabilities be denoted by

$$
S_{k}=\sum_{i=1}^{r} I_{(0,1]}\left(p_{i, k}^{\prime}\right)
$$

the recursion terminates when $s_{k}=s_{k-1}$. The final $p_{1, k}^{\prime}, \ldots, p_{r, k}^{\prime}$ are then the normalized forecast probabilities. 


\section{7 | EXTREME VALUE}

\section{TRANSFORMATION (EVT)}

Baron, Mellers, Tetlock, Stone, and Ungar (2014) establish the fact that extremizing forecasts helps in reducing the Brier score of forecasts. A transformation proposed in Ramos (2014) has been used to great effect. This method works for any number, $r$, of answer categories.

Let $p_{1}, \ldots, p_{r}$ be raw aggregated forecasts. First, we check that all forecasts are in the interval $[0,1]$. Suppose $r^{*}$ forecasts are greater than zero, $1 \leq r^{*} \leq r$. We will call these forecasts "relevant forecasts." Ignoring the nonrelevant forecasts categories, there are now $p_{1}^{\prime}, \ldots, p_{r^{*}}^{\prime}$ forecasts. The EVT is

$$
\operatorname{EVT}\left(p \prime_{k}\right)= \begin{cases}\psi_{1}\left(\frac{p \prime_{k}}{\psi_{1}}\right)^{v} & \text { for } p_{k}<1 / r^{*}-\delta, \\ p_{k} & \text { for } p \prime_{k} \in\left[1 / r^{*}-\delta, 1 / r^{*}+\delta\right], \\ 1-\psi_{2}\left(\frac{1-\prime_{k}}{\psi_{2}}\right)^{v} & \text { for } p_{k}>1 / r^{*}-\delta,\end{cases}
$$

where $\psi_{1}=1 / r^{*}-\delta, \psi_{2}=1-\left(1 / r^{*}+\delta\right), \delta$ is the chosen radius of the neutral area, and $v$ is the chosen parameter for degree of extremization (see Ramos, (2014). Finding the appropriate values for $\delta$ and $v$ was done by training over a grid of values for 100 random IFPs from years $1-2$ of the GJP. Training over these IFPs gave us values of $\delta=0.1$ and $v=2$. Figure 9 shows the EVT function for $r^{*}=2$ and 3 .

Values in the "neutral radius" around $1 / r^{*}$ are not changed, where the parameter $\delta$ controls the radius. Forecasts outside of the "neutral" radius about $1 / r^{*}$ are moved closer to one of the extreme values of either 1 or 0 depending on whether the initial value is above or below $1 / r^{*}$.

\section{ALTERNATIVE METHODS}

Several variations on the above methods were also tried. One seemingly promising one was the introduction of a collection of $m$ nonconstant weight vectors, each of length $n$ applied to the forecasts, into the partial Fourier $L_{2} E$ regression. The modified criterion is the weighted sum of these $m$ different partial $L_{2} E$ fits, namely:

$$
L_{W}(\sigma, w, \epsilon)=\sum_{k=1}^{m} v_{k}\left[\frac{w^{2}}{2 \sqrt{\pi \sigma^{2}}}-2 w\left\langle W_{k}, \phi\left(\underline{\epsilon} \mid 0, \sigma^{2}\right)\right\rangle\right] .
$$

Here $m$ is the number of different weighting schemes, $w$ is the partial $L_{2} E$ parameter, $W_{k}$ s are weight vectors summing to 1 , and $v_{k}$ is the weight of the $k$ th $L_{2} E$ criterion. The inner product notation, $\left\langle W_{k}, \phi\right\rangle$, generalizes the equally weighted second sum in Equation 4. While this method maintains the unbiasedness of the estimator of $E[f(X)]$, the estimator is no longer the best (in terms of mean squared error) unbiased linear estimator if $W_{k} \neq \frac{1}{n}$ for some $k$. However, no nontrivial combination of weights tried returned a result with lower mean Brier score than the method proposed earlier.

We also experimented with Bayesian methods, but because a single point forecast is required for each day, additional sources of error are introduced through point selection for the aggregated forecast. A popular method is to choose the mode of the posterior distribution as the point forecast but this and other Bayesian point forecasts, such as the mean and median of the posterior distribution, were found to be inferior to our frequentist approach in terms of mean Brier score.

Additionally, the extremization proposed by Satopää, Pemantle, and Ungar (2015) was reevaluated, but with slightly worse results than Equation 9. Also, usual aggregation by weighting via inverse $\sigma^{2}$ or MSE is not likely to work in a robust setting, where groups of forecasts are essentially ignored. This is because different robust methods could choose to "ignore" different sets of data, which may lead to the chosen aggregate forecast being outside of the desired range.

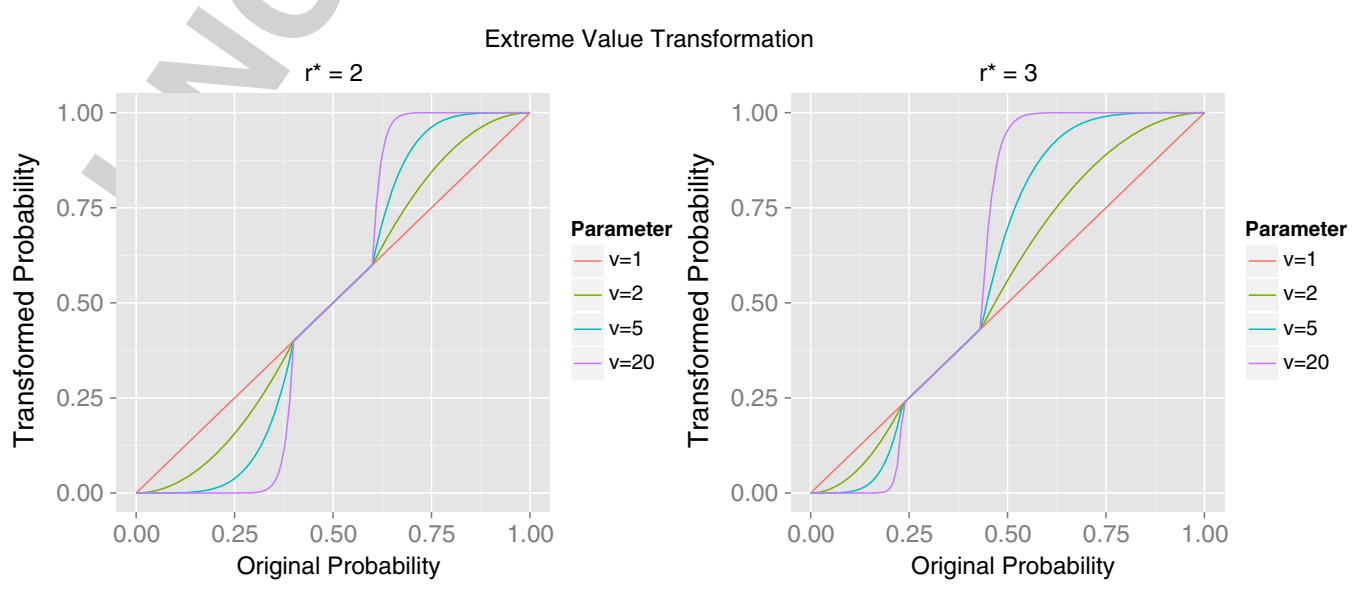

FIGURE 9 Robust extreme value transformation from Equation 9 with $\delta=0.1$ [Colour figure can be viewed at wileyonlinelibrary.com] 


\section{9 | RESULTS}

While individually the methods described above may decrease the mean Brier score by seemingly small amounts, when performed in sequence the net gain is substantial. There were several baseline aggregators used by the GJP that we can immediately compare to our aggregator: the daily top individuals median; the Inkling Control aggregator given on the GJP web page (this is the baseline aggregator to which all others are compared); and the daily mean. The original $L_{2} E$ regression aggregator by Ramos (2014) is also compared as well as Super Teams Logit, which had the lowest mean Brier score for year 4 . These scores were computed by the GJP software over the period 2013-07-08 to 2015-06-09, and represent the average of Brier scores for 283 individual IFPs over that period. Figure 10 shows that the Fourier $L_{2} E$ aggregator and the original $L_{2} E$ aggregator are both notably more concentrated at the origin than the other listed aggregators. This

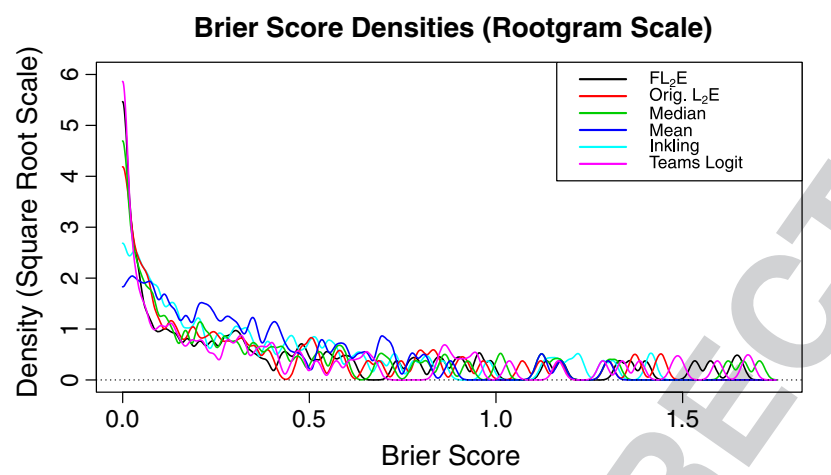

FIGURE 10 Density plots of all six aggregators for years 3 and 4 ( $n=283$ ) [Colour figure can be viewed at wileyonlinelibrary.com] explains the higher standard deviation and smaller mean given in the Table 1.

Ranking on the mean Brier score gives Fourier $L_{2} E$ an edge over the previous $L_{2} E$ forecasting method (Top Indivs $L_{2} E$ ), while both $L_{2} E$ algorithms outperform the median and mean aggregators as well as Inkling Control. Taking a log transform of the individual IFP Brier scores and performing a paired $t$-test gives a $p$-value of 0.033 , rejecting equal means for Top Indivs $L_{2} E$ and Fourier $L_{2} E$. In conclusion, although the standard deviation of Fourier $L_{2} E$ is larger than that of Top Indivs $L_{2} E$, IARPA and the GJP measured the quality of aggregation by the mean Brier score rather than its standard deviation or mean squared error. The Fourier $L_{2} E$ 's mean was statistically significantly reduced. We note that decreasing the mean Brier score is a nontrivial accomplishment in its own right.

\section{$10 \mid$ DISCUSSION}

Many different tools were experimented with in order to lower the mean Brier score of our Fourier $L_{2} E$ regression method. Robustness was the largest factor in this reduction. Using top forecasters, a variance-stabilizing transformation, and random starting values were also instrumental in reducing mean Brier score. Also, the truncated Fourier series regression structure as opposed to a simpler regression model, such as a polynomial model, let the regression follow more complicated and unpredictable trends of geopolitical forecasting. The combination of all methods shown in this paper, aside from the Fourier $L_{2} E$ regression itself, reduced the mean Brier score from 0.164 to 0.155 , a highly significant difference, both statistically and practically.

TABLE 1 Method results for years 3 and 4

\begin{tabular}{llll} 
Method & Mean Brier score & Brier score SD & root MSE \\
\hline Inkling Control & 0.2100 & 0.2760 & 0.3468 \\
Top Indivs Mean & 0.2319 & $\mathbf{0 . 2 2 5 4}$ & 0.3234 \\
Top Indivs Median & 0.1632 & 0.2912 & 0.3338 \\
Top Indivs $L_{2} E$ & 0.1609 & 0.2734 & $\mathbf{0 . 3 1 7 2}$ \\
Top Indivs Fourier $L_{2} E$ & 0.1551 & 0.3049 & 0.3420 \\
Super Teams Logit & $\mathbf{0 . 1 5 1 9}$ & 0.3137 & 0.3486 \\
\hline
\end{tabular}

TABLE 2 Median method results for years 3 and 4

\begin{tabular}{llll} 
Method & Median & Median absolute deviation & Sum \\
\hline Inkling Control & 0.0946 & 0.1063 & 0.2010 \\
\hline Top Indivs Mean & 0.1684 & 0.1719 & 0.3403 \\
\hline Top Indivs Median & 0.0447 & 0.0620 & 0.1067 \\
Top Indivs $L_{2} E$ & 0.0463 & 0.0570 & 0.1032 \\
\hline Top Indivs Fourier $L_{2} E$ & 0.0254 & 0.0356 & 0.0610 \\
\hline Super Teams Logit & $\mathbf{0 . 0 1 7 8}$ & $\mathbf{0 . 0 2 5 6}$ & $\mathbf{0 . 0 4 3 4}$ \\
\hline
\end{tabular}


Areas of further research include incorporating covariates of the subjects and their forecasts. Further theoretical investigations into the justifications of the various extreme value transformation would be useful. Finally, graphical and web-based presentation of aggregate forecasts were partially investigated but discontinued at IARPA's request. We believe this would be useful to look at again.

\section{ACKNOWLEDGEMENTS}

This research was supported by the Intelligence Advanced Research Projects Activity (IARPA) via the Department of Interior National Business Center (DoI/NBC) contract number D11PC20061. The US Government is authorized to reproduce and distribute reprints for Government purposes notwithstanding any copyright annotation thereon. Disclaimer: The views and conclusions expressed herein are those of the authors and should not be interpreted as necessarily representing the official policies or endorsements, either expressed or implied, of IARPA, DoI/NBC, or the US Government.

\section{ORCID}

Daniel Cross (D) http://orcid.org/0000-0003-1574-0194

\section{REFERENCES}

Anscombe, F. J. (1948). The transformation of Poisson, binomial and negative-binomial data. Biometrika, 35, 246-254.

Baron, J., Mellers, B. A., Tetlock, P. E., \& Stone, E. (2014). Ungar, L. H. Two reasons to make aggregated probability forecasts more extreme. Decision Analysis, 11(2), 133-145.

GJP (2015a). Aggregation methods. Retrieved from. http://gordo. seas.upenn.edu/methods

GJP (2015b). The Good Judgment Project. Retrieved from http:// www.goodjudgmentproject.com

Kirkpatrick, S., Gelatt, C. D., \& Vecchi, M. P. (1983). Optimization by simulated annealing. Science, 220(4598), 671-680.

Mellers, B. A., Ungar, L., Baron, J., Ramos, J., Gurcay, B., Fincher, K., \& ...Tetlock, P. E. (2014). Psychological strategies for winning a geopolitical forecasting tournament. Psychological Science, 25, 1106-1115.

Ramos, J. (2014). Robust Methods for Forecast Aggregation (Doctoral Thesis). Houston, TX: Rice University.

Satopää, V. A., Pemantle, R., \& Ungar, L. H. (2015). Modeling probability forecasts via information diversity. Manuscript under review.

Scott, D. W. (2001). Parametric statistical modeling by minimum integrated square error. Technometrics, 43, 274-285.

Scott, D. W. (2004). Partial mixture estimation and outlier detection in data and regression. In Hubert, M., Pison, G., Struyf, A., \& Van Aelst, S. (Eds.), Theory and applications of recent robust methods. Berlin, Germany: Springer, pp. 297-306.

Tetlock, P. (2005). Expert political judgment: How good is it? How can we know? Princeton, NJ: Princeton University Press.

Tetlock, P., \& Gardner, D. (2015). Superforecasting: The art and science of prediction. New York, NY: Crown.
Ungar, L., Mellors, B., Satopää, V., Baron, J., Tetlock, P., Ramos, J., \& Swift, S. (2012). The Good Judgment Project: A large scale test (AAAI Technical Report, FS-12-06). Palo Alto, CA.

\section{Daniel Cross}

Jaime Ramos

Barbara Mellers

Philip E. Tetlock $\square$

David W. Scott $\square$

How to cite this article: Cross D, Ramos J, Mellers B, Tetlock PE, Scott D W. Robust Forecast Aggregation: Fourier L2E Regression. J Forecast. 2017;1-10. https://doi.org/10.1002/for.2489 